\title{
Conduite De Changement Organisationnel Dans Le Secteur De Santé Marocain. Cas Du Centre Hospitalier Regional D'agadir
}

\author{
Rachid Zammar \\ Professeur Habilité à la faculté des sciences de Rabat. \\ Université Mohammed V. Maroc \\ Noureddine Abdelbaki \\ Professeur Habilité à la faculté des sciences juridiques \\ économiques et sociales d'Agadir. Université Ibn Zohr. Maroc
}

doi: 10.19044/esj.2016.v12n27p109 URL:http://dx.doi.org/10.19044/esj.2016.v12n27p109

\begin{abstract}
This article proposes a clarification of driving organizational change in the Moroccan health sector and levers used to set up and considers the following question: how the establishment of hospital project (EHP) proves as tool driving change in the Moroccan health sector? The methodology is based on an exploratory study among the staff of a particular hospital which is the regional hospital of Agadir (CHRA). The main conclusion of the study is that the implementation of the EHP, at the CHRA enabled the upgrade of the hospital. However, alone man cannot make the change, the financial and technical resources have a clear role in improving care services to the population.
\end{abstract}

Keywords: Change, communication, hospital center, resistance

Résumé

Cet article propose un éclaircissement de la conduite du changement organisationnel dans le secteur de santé marocain et les leviers utilisés pour le mettre en place et s'assigne comme objectif d'examiner la question suivante: comment le projet d'établissement hospitalier ( $\mathrm{PEH}$ ) s'avère comme outil de conduite de changement dans le secteur de santé marocain? La méthodologie adoptée repose sur une étude exploratoire menée auprès du personnel d'un hôpital particulier qui est le centre hospitalier régional d'Agadir (CHRA). La principale conclusion de l'étude est que l'implantation du $\mathrm{PEH}$, au niveau du CHRA a permis la mise à niveau de 1'hôpital. Cependant, à lui seul 1'Homme ne peut pas assurer le changement, 
les ressources financières et techniques ont un rôle bien défini dans l'amélioration des services de soins offerts à la population.

Mots clés: Changement, communication, centre hospitalier, résistance

\section{Introduction}

Au début des années 1990, la question hospitalière au Maroc est devenue préoccupante pour les pouvoirs publics. La part des dépenses hospitalières dans la consommation médicale qui ne cesse d'augmenter, l'amélioration du mode de financement et l'expérimentation d'une comptabilité analytique sont autant d'éléments de changement pour qualifier l'hôpital marocain.

En 1996, la méthode de management hospitalier à travers le projet d'établissement hospitalier $(\mathrm{PEH})$ était la première réflexion au Maroc lancée au centre hospitalier et universitaire (CHU) de Rabat. Toutefois, ce n'est qu'en 2001 avec le lancement de la « réforme hospitalière », que le ministère de la santé a prévu la mise en place du PEH dans cinq hôpitaux publics pilotes : Agadir, Béni Mellal, Meknès, Safi et Settat.

Ce PEH aurait dû déboucher, à travers la mobilisation des ressources de l'hôpital, dans un sens partagé par tous et adapté aux caractéristiques de son environnement, sur la mise en œuvre d'actions cohérentes et programmées.

Cette recherche qui est en partie exploratoire s'assigne comme objectif de traiter de la problématique suivante: comment le projet d'établissement hospitalier s'avère comme outil de conduite de changement dans le secteur de santé Marocain? Notre hypothèse est que la mise en œuvre $\mathrm{du} \mathrm{PEH}$ est une dynamique de changement, bien conduite, peut permettre l'amélioration de la performance de l'hôpital.

\section{Revue de littérature}

A.Guilhon (1998, p.98) propose la définition suivante du vocable changement organisationnel: «Le changement organisationnel désigne toute modification dans les structures et/ou les comportements des membres de l'organisation résultant d'une adaptation à l'environnement ou d'une action volontaire du dirigeant en vue d'assurer le maintien ou la croissance de l'entreprise dans son environnement ».

La notion du changement se présente comme une modification apportée à l'évolution de la structure, aux modes de gestion, à la technologie, à la transformation des dimensions culturelle et humaine de l'organisation (croyances, valeurs, règles) ainsi qu'aux personnes qui y travaillent en vue de l'amélioration de son fonctionnement et de sa performance. 


\section{La méthodologie adoptée}

La démarche adoptée repose sur un positionnement épistémologique déterminé en grande partie par la nature exploratoire de notre étude qui nous amène à adopter une posture interprétativiste. Cette posture va nous aider à comprendre comment le projet d'établissement hospitalier s'avère comme outil de conduite de changement dans le secteur de santé marocain en s'intéressant au cas particulier du centre hospitalier régional d'Agadir (CHRA) à travers une enquête menée auprès de 51 personnes travaillant au sein de cet hôpital.

\section{Résultat}

Pour analyser les leviers de la conduite de changement organisationnel, on a choisi trois fonctions essentielles : la gestion financière, la gestion des ressources humaines et la gestion du matériel et des équipements.

\section{La gestion financière}

L'allocation des ressources aux hôpitaux s'effectue sur la base de budgets programmes établis pour une période pluriannuelle définissant les objectifs, les moyens et les résultats attendus. Le budget programme est établi par référence au projet d'établissement hospitalier dûment approuvé par le ministre de la santé.

Tableau $\mathrm{n}^{\circ} 1$. Structure de la trésorerie

\begin{tabular}{|c|c|c|c|}
\hline RESSOURCES & 2005 & 2006 & 2007 \\
\hline $\begin{array}{c}\text { Excédent de l'exercice } \\
\text { écoulé (N-1) }\end{array}$ & 2580567,89 & 1912081,36 & 3529596,38 \\
\hline Recettes réalisées au & 8873077,53 & 11893742,94 & 9869836,55 \\
\hline Subvention de l'État & 3038000,00 & 2600000,00 & 2600000,00 \\
\hline TOTAL & 14491645,42 & 16405824,30 & 15999432,93 \\
\hline DÉPENSES (PAIEMENT) & - & - & - \\
\hline Personnel & 600,00 & 600,00 & 600,00 \\
\hline Matériel et dépenses diverses & 12578964,06 & 12875627,92 & 14541054,07 \\
\hline TOTAL & 12579564,06 & 12876227,92 & 14541654,07 \\
\hline DISPONIBLE (N+1) & 1912081,36 & 3529596,38 & 1457778,86 \\
\hline
\end{tabular}

Source : Centre hospitalier régional d'Agadir (CHRA)

La trésorerie du centre hospitalier régional d'Agadir a été excédentaire pour les années 2005, 2006 et 2007.

\section{La gestion des ressources humaines}

Le CHRA dispose de 531 personnes comme ressources humaines, subdivisé en différentes catégories de personnel : une centaine de médecins, plus de 300 infirmiers, une quarantaine de personnel administratif ainsi que 
60 agents sociaux (l'ensemble de personnel qui s'occupent des activités d'hygiène et de restauration)

Le pôle administratif qui a pour rôle d'appuyer, soutenir et accompagner tout le personnel de l'hôpital, se subdivise à son tour en plusieurs unités : financière, de communication, de matériel et équipement.

En effet, parmi les grands changements qu'a connus le CHRA et comme apport de la réforme hospitalière, est la création d'une unité de gestion des ressources humaines en janvier 2004 qui n'existait pas auparavant.

Tableau n². Répartition de l'effectif par catégorie professionnelle

\begin{tabular}{|c|c|c|c|c|}
\hline Effectifs & Homme & Femme & Total & Pourcentage \\
\hline Médecins & 64 & 35 & 99 & $18,64 \%$ \\
\hline Infirmiers & 145 & 187 & 332 & $62,52 \%$ \\
\hline Administratifs & 21 & 17 & 38 & $7,16 \%$ \\
\hline Soutiens & 40 & 22 & 62 & $11,68 \%$ \\
\hline Total & 270 & 261 & 531 & $100,00 \%$ \\
\hline Pourcentage & $50,85 \%$ & $49,15 \%$ & $100,00 \%$ & \\
\hline
\end{tabular}

Source : Centre hospitalier régional d'Agadir (CHRA)

En effet, cette répartition du personnel de l'hôpital signifie une si riche expérience, surtout pour les médecins, exploitée et transmise aux jeunes. Mais aussi, une phase de fatigue et de faiblesse de la capacité physique. Ainsi, l'âge moyen présent dans l'hôpital est de 44 ans et 5 mois.

\section{La communication au sein du CHRA}

La communication est l'un des leviers développés dans le cadre du PEH, pour cela le CHRA a crée une unité de communication dont le rôle est de gérer et organiser la communication interne et externe de l'hôpital et qui organise des plans de communication en s'adaptant aux besoins de l'hôpital et en se référant à son actualité. Plusieurs types de communication, écrite, orale et visuelle sont utilisés (les notes d'information, les notes de service, les réunions périodiques, le journal de l'hôpital, internet et dernièrement l'intranet qui vient d'être installée).

Toutefois, reste à savoir, si tous les acteurs de l'hôpital sont sujets de cette communication apparente riche ? Est ce qu'ils utilisent l'internet ou l'intranet pour partager les informations aussi bien internes qu'externes ? L'hôpital Hassan est le premier hôpital où s'est développé le pôle de système d'information et d'informatisation dans le cadre de PEH.

L'enquête menée auprès du personnel du CHRA a permis de répondre à ces questions.

Contrairement à la direction qui a déclaré une présence forte de communication et de ses moyens, le personnel interrogé au niveau de l'enquête menée, n'apprécie pas la communication au sein de cette 
organisation et c'est ce qu'on a constaté en analysant le questionnaire déjà établi et qui nous a donné un pourcentage de $71.2 \%$ de personnel qui déclare l'absence de la communication entre tout le personnel au sein de l'hôpital.

\section{Les réunions}

D'après l'administration du CHRA, ce dernier organise chaque quinzaine ou encore quand il est nécessaire des réunions. Ainsi il y a une intégration de tout le comité de direction et d'autres acteurs choisis dans le projet d'établissement, par exemple le responsable du matériel médical dans le cadre de l'application du management participatif et l'implication du personnel dans les décisions prises, mais le problème rencontré à ce niveau réside dans l'absence et la non assistance à ces réunions par la plupart des acteurs du service médical et infirmiers.

Concernant, la participation du personnel aux réunions, on remarque bien que la plupart du personnel affirme la non participation aux réunions avec un pourcentage de $69.2 \%$ contre $28.8 \%$ qui y participent. Bien entendu que la plupart des participants à ces réunions appartiennent au service administratif ce qui est considéré comme une faille témoignant de la faible communication interne et un indicateur de résistance au changement.

\section{Le système d'information informatisé}

Le CHRA est le seul au Maroc qui a intégré un système d'information informatisé établi par une compagnie espagnole dans le cadre d'un projet lancé par le ministère de la santé. Ce système permet le contact entre les différents services ainsi qu'avec le ministère lui-même et qui constitue aussi un moyen de contrôle, de communication et d'information transparente (toutes les opérations chirurgiennes d'un médecin, les informations sur un patient: date d'entrée, bloc, médecin,...). Mais ce système reste encore non appliqué au niveau de tout l'hôpital puisqu'il n'est pas encore utilisé par l'ensemble des acteurs concernés et puisque la résistance au changement par le personnel est encore présente à ce niveau.

\section{La formation}

En se référant aux besoins de l'hôpital en matière de formation de son personnel, l'administration s'occupe d'établir un plan de formation répondant à ses besoins en termes de qualité et de quantité. Ce plan est adressé à la délégation régionale de la santé qui à son tour le transmet au ministère de la santé qui s'occupe de la mise en œuvre et la fourniture des moyens nécessaires pour son application. Mais, malheureusement, ce plan n'est pas mis en pratique du fait du manque du budget et que la décision de la formation relève du central. 


\section{La satisfaction du personnel}

Pour la satisfaction du personnel au niveau des conditions de travail, on a pu ressortir les résultats suivants :

- Sur 52 personnes interrogées, 2.19 est la moyenne de la satisfaction au niveau des conditions de travail ;

- 42.3\% du personnel ne sont plutôt pas satisfaits des conditions de travail au sein du CHRA, 32.7\% sont plutôt satisfaits, $21.2 \%$ sont pas du tout satisfaits et $3.8 \%$ sont tout à fait satisfaits.

\section{La gestion du matériel et de l'équipement}

$51.9 \%$ du personnel déclarent qu'il y a une insuffisance du matériel, 40.4\% affirment que le matériel est disponible en grande partie et enfin 7.7 $\%$ disent qu'ils disposent de la totalité du matériel et des équipements nécessaires pour le bon déroulement de leur travail.

Concernant les problèmes rencontrés par le personnel au niveau des locaux de leur travail : 22 personnes de l'échantillon interrogé déclarent l'inadaptation des locaux à leurs besoins, 10 personnes le trouvent trop étroit, 9 personnes ont parlé de l'existence d'un matériel informatique dépassé, et enfin 8 personnes ont déclaré que les locaux sont anciens et que le matériel est usagé, alors que 8 d'autres ont parlé de la chaleur excessive ressentie au niveau de ces locaux.

\section{La certification}

Après avoir obtenu le deuxième prix en matière d'application de la réforme en 2007 dans la mise en œuvre du PEH, le CHRA a décidé d'introduire le processus d'accréditation hospitalière en 2009 en agissant sur quatre dimensions : stratégique, culturelle, technique et structurelle.

Toutefois, concernant les principes du concours qualité l'accent a été mis sur : le processus, le système dans sa globalité, le volontariat, l'effort et la dynamique d'amélioration.

Quant aux dimensions prises en considération pour le concours qualité, elles sont comme suit: la satisfaction (de la communauté, du personnel), l'éthique (de l'établissement, du personnel), l'accessibilité/disponibilité, la continuité, la rationalisation des ressources, la sécurité/ la réactivité, le leadership/ le partenariat, l'amélioration continue, les compétences techniques et la fonctionnalité.

\section{Discussions}

Durant les 5 ans programmés dans le cadre du projet d'établissement apporté par la réforme, le CHRA a pu bénéficier de plusieurs améliorations qu'on peut classer en deux volets : 
$\Rightarrow \quad$ le premier concerne tout ce qui est matériel et équipements, la construction de complexe des urgences, le complexe de réanimation, la nouvelle pédiatrie et également l'acquisition d'un matériel informatique, médical et médico-technique ;

$\Rightarrow \quad$ le deuxième porte sur les outils de gestion implantés notamment l'unité de gestion des ressources humaines et un système de comptabilité (gestion financière et comptabilité analytique) amélioré et informatisé afin d'améliorer les capacités de gestion de l'hôpital.

Le personnel du CHRA est âgé et incarné par les anciennes pratiques face à une administration apportant de nouvelles pratiques, de nouveaux changements et travaillant sous l'encadrement d'une assistance technique.

Généralement, $57.7 \%$ du personnel enquêté n'est pas informé de la mise en œuvre d'une réforme hospitalière par manque de communication afin d'impliquer le personnel, ce qui entrave à fort degré la réalisation des objectifs visés par la réforme.

Les problèmes les plus rencontrés au niveau de l'hôpital résident dans la gestion des ressources humaines. La résistance au changement, surtout au niveau du personnel infirmier et médecins, s'est manifestée depuis la réforme, et ce malgré toutes les actions menées et accompagnant la réforme, tels que la formation locale et externe, la sensibilisation, les réunions, l'assistance des canadiens qui s'occupaient du suivi de l'application de la réforme et également de son contrôle.

Egalement, il y a une insuffisance du personnel en profil et en quantité au minimum plus de 100 postes sont vacants, mais le problème réside toujours dans la dépendance vis-à-vis du ministère de la santé qui s'occupe également du recrutement,

Pour assurer le succès du changement organisationnel du CHRA, il faut lui doter d'une ressource humaine bien qualifiée, notamment un corps médical qui se montre créatif et dont la compréhension et la maitrise ne s'étale pas seulement au domaine médical mais également au domaine managérial. Dans ce sens une administration avec des profils de gestionnaire reste souhaitable à la place des postes de décision qui sont occupés par des techniciens responsables. Tout le personnel doit être bien choisi et bien formé et informé sur tous les organes de l'hôpital, sa stratégie, ses objectifs et ses finalités. La création d'un milieu de travail adapté avec un esprit relationnel professionnel et une stabilité des responsables peut impliquer tout le personnel dans le changement et réduire la résistance à la réforme.

L'implantation du PEH, au niveau du CHRA a permis plusieurs réalisations parmi lesquelles la mise à niveau de l'hôpital. Les investissements concernant les services de soutien réalisés à $100 \%$. Et si la communication a été insuffisante, la formation n'a concerné que quelques responsables. 
Le leadership du directeur n'est pas reconnu par tout le personnel, l'insuffisance du financement et les contraintes techniques ont contribué à la non réalisation de quelques investissements, cela n'explique que la mauvaise gouvernance que connait les hôpitaux publics et la résistance du personnel qui veut rester dans une situation de sous emploi et maintenir l'exploitation d'une organisation à $50 \%$ contre $100 \%$ dans les cliniques privés. Et c'est dans ce sens que le ministère a investi dans la réforme hospitalière pour une mise à niveau des hôpitaux tout en envisagent un changement organisationnel et porter concurrence au secteur privé.

Toutefois, à lui seul l'Homme ne peut pas assurer le changement, les ressources financières et techniques ont un rôle bien défini dans l'amélioration des services de soins offerts à la population.

\section{Conclusion}

L'objectif du projet de modernisation des hôpitaux et de leur management a été atteint. Des acquis ont pu être obtenus, le résultat était satisfaisant malgré les contraintes liées à la mise en œuvre. Le CHRA était l'un des hôpitaux pilotes qui a connu la mise en œuvre de plusieurs pôles de gestion notamment la gestion financière et comptable, le système d'information et d'informatisation, et le plan directeur immobilier (PDI). Les résultats qui ont émané de ce projet ont tous fait l'objet d'une réglementation et d'une généralisation de façon systématique comme l'organisation des hôpitaux au Maroc.

\section{References:}

ACODESS : Guide en organisation hospitalière : Elaborer et mettre en œuvre un projet d'établissement hospitalier dans les pays en développement (coordonnateur Cyrille Callens), version corrigée 06/03/2010, 132p.

Audet M. (2009). Vision, planification et évaluation: les conditions clés d'un changement, Revue Gestion, vol 34, p.55-63.

Autissier D. et Moulot J.-M. (2003). Pratique de la conduite du changement, Paris, Dunod.

Giroux, N. (1991). La gestion du changement stratégique, Gestion, mai, p 814.

Lahbous M, Zammar R. (2011). Management des organisations : fondements théoriques, principes, tâches et méthodes, Rabat, Dar Al Kalam.

Luyan J-P. (2010). Le changement sans stress : dépasser les résistances et la pression, Paris, Éditions d'Organisation.

Meier O. (2007). Gestion du changement, Paris, Dunod. 